interferon- $\gamma$ response, and myeloid inflammatory gene-expression signatures may help to predict response to inhibitors of VEGF, PD-1, and PD-L1. Previously, it had been suggested that PD-L1 positivity might also predict the increased efficacy of immune checkpoint inhibitors. ${ }^{8}$ Although the tests used to assess positivity (and thus the number of positive tumors) were very different in each of the three trials, the rate of efficacy of nivolumab plus ipilimumab in PD-L1-positive metastatic renal-cell carcinoma was very high and will have to be confirmed prospectively. In contrast, the efficacy rate of this same combination among favorable-risk patients was disappointing. These observations were not reported in the two trials now reported in the Journal.

Disclosure forms provided by the author are available with the full text of this editorial at NEJM.org.

From the Gustave Roussy Cancer Campus, Villejuif, France.
1. Motzer RJ, Hutson TE, Tomczak P, et al. Sunitinib versus interferon alfa in metastatic renal-cell carcinoma. $\mathrm{N}$ Engl J Med 2007;356:115-24.

2. Motzer RJ, Escudier B, McDermott DF, et al. Nivolumab versus everolimus in advanced renal-cell carcinoma. $\mathrm{N}$ Engl J Med 2015;373:1803-13

3. Motzer RJ, Tannir NM, McDermott DF, et al. Nivolumab plus ipilimumab versus sunitinib in advanced renal-cell carcinoma. N Engl J Med 2018;378:1277-90.

4. Motzer RJ, Penkov K, Haanen J, et al. Avelumab plus axitinib versus sunitinib for advanced renal-cell carcinoma. N Engl J Med 2019;380:1103-15.

5. Rini BI, Plimack ER, Stus V, et al. Pembrolizumab plus axitinib versus sunitinib for advanced renal-cell carcinoma. $\mathrm{N}$ Engl J Med 2019;380:1116-27.

6. Rini BI, Melichar B, Ueda T, et al. Axitinib with or without dose titration for first-line metastatic renal-cell carcinoma: a randomised double-blind phase 2 trial. Lancet Oncol 2013;14:1233-42. 7. McDermott DF, Huseni MA, Atkins MB, et al. Clinical activity and molecular correlates of response to atezolizumab alone or in combination with bevacizumab versus sunitinib in renal cell carcinoma. Nat Med 2018;24:749-57.

8. Topalian SL, Hodi FS, Brahmer JR, et al. Safety, activity, and immune correlates of anti-PD-1 antibody in cancer. $\mathrm{N}$ Engl J Med 2012;366:2443-54.

DOI: 10.1056/NEJMe1900887

Copyright (c) 2019 Massachusetts Medical Society.

\title{
Psychosis during Attention Deficit-Hyperactivity Disorder Treatment with Stimulants
}

\author{
Samuele Cortese, M.D., Ph.D.
}

Attention deficit-hyperactivity disorder (ADHD) is one of the most commonly diagnosed conditions in child and adolescent mental health services. Stimulants, including methylphenidate and amphetamines, are recommended for ADHD treatment. ${ }^{1}$ Methylphenidate is the most frequently prescribed stimulant in many countries; however, data from private insurance claims show that amphetamines are more commonly prescribed in the United States. ${ }^{2}$ Despite meta-analyses that show the efficacy of stimulants in reducing ADHD symptoms, ${ }^{3}$ at least in the short term, the quality of evidence and the safety of these medications continue to be debated. In particular, psychosis can occur during stimulant treatment and can be traumatic for patients and their families.

Until now, there have been very few data on the comparative risk of psychosis during treatment with methylphenidate and amphetamines. The study by Moran et al. ${ }^{4}$ in this issue of the Journal is a contribution to this literature. The au- thors analyzed data from two national U.S. insurance claims databases that included 337,919 adolescents and young adults, 13 to 25 years of age, who received a prescription for a stimulant for ADHD from January 2004 through September 2015. Patients who received methylphenidate were compared with those who received amphetamine with the use of propensity-score matching, which took into account broad sociodemographic and psychiatric characteristics, and the incidence of psychotic episodes that occurred during follow-up were compared between the two stimulant groups. During a median follow-up period of 4 to 5 months, new-onset psychotic episodes for which antipsychotic medications were prescribed were uncommon but nevertheless occurred in approximately 1 in 660 patients. The percentage of patients who had an episode of psychosis (defined as a new diagnosis code for psychosis and a prescription for an antipsychotic medication) was significantly higher in the amphetamine group than in the 
methylphenidate group $(0.21 \%$ vs. $0.10 \%)$, and psychotic episodes occurred a median of 128 days after exposure to the medication. These findings are consistent with a meta-analysis of randomized trials ${ }^{3}$ in that they suggest a more favorable safety profile for methylphenidate than for amphetamine in young patients, at least at the group level. ${ }^{3}$

The findings of the current study should not be considered definitive. Observational studies such as this one can provide information on uncommon adverse events in real-world clinical practice that are challenging to assess in randomized trials performed over brief periods. However, even sophisticated approaches, such as the ones used in this study to address possible biases, do not have the advantages of randomized trials in excluding confounding factors. ${ }^{5}$

Furthermore, the study cannot establish causality. A previous report involving 20,568 patients did not support a causal role of methylphenidate in psychosis. ${ }^{6}$ That analysis used a self-controlled case-series design, whereby the risk of psychosis when participants were taking medication was compared with the risk when they were not taking medication, in order to reduce confounding by indication. It has been proposed that there are persons who have "low vulnerability" (in whom psychosis will rarely develop even after exposure to stimulants) and persons who have "high vulnerability" (who are likely to present with psychosis after taking low doses of a stimulant or even after no exposure to stimulants). ${ }^{7}$ Therefore, whether psychosis is due to stimulant use, to inherent vulnerability to psychosis, or to the interaction of those two factors remains unclear. In this regard, an intriguing finding from post hoc analyses of the current study was that the difference in the risk of psychosis between drugs was not present when medications were prescribed by psychiatrists as compared with other physicians. A possible interpretation is that psychiatrists more readily detected prodromal psychotic features that increase the risk of treatmentrelated psychosis, and they avoided the prescription of amphetamine in such cases.

Despite uncertainties regarding causal mechanisms, the study by Moran and colleagues provides important data on the incidence of psychosis observed in routine practice among patients with ADHD. These figures could inform decision making among patients, families, and physicians when stimulants are prescribed for ADHD, and a balance is desirable between the safety and the effectiveness of a drug for ADHD core symptoms. The ostensible benefits of stimulants with respect to problems related to ADHD (such as a reduction in criminality, as suggested in a previous study ${ }^{8}$ ) should also be considered before treatment is initiated.

It was beyond the scope of the current study to comment on the management of psychotic events during stimulant treatment. Analyses of Food and Drug Administration data and case reports ${ }^{9}$ have shown that psychotic symptoms are short-lived and resolve after discontinuation of the stimulant in $92 \%$ of patients, even without treatment with antipsychotic medications. Guidelines for the subsequent treatment of ADHD include the option of a cautious rechallenge with stimulants after an episode of stimulant-associated psychosis. ${ }^{1}$ Although rechallenge was not assessed in the study by Moran and colleagues, their findings could suggest that methylphenidate is a safer option than amphetamine for a rechallenge, at least in patients in the age groups studied. This possibility, and the effects of rechallenge with stimulants in patients whose psychotic episodes have stabilized with antipsychotic medications, deserve additional investigation.

Currently, it is not possible to predict which patients will have psychotic episodes after stimulant treatment. Perhaps techniques such as machine learning applied to large data sets from randomized trials, combined with observational data, ${ }^{10}$ will provide predictors at the individual patient level.

Disclosure forms provided by the author are available with the full text of this editorial at NEJM.org.

From the Center for Innovation in Mental Health, Academic Unit of Psychology, Faculty of Environmental and Life Sciences, and Clinical and Experimental Sciences, Faculty of Medicine, University of Southampton, and Solent NHS Trust, Southampton - all in the United Kingdom.

1. Attention deficit hyperactivity disorder: diagnosis and management. NICE guideline [NG87]. London: National Institute for Health and Care Excellence (NICE), March 2018 (https:// www.nice.org.uk/guidance/NG87).

2. Raman SR, Man KKC, Bahmanyar S, et al. Trends in attention-deficit hyperactivity disorder medication use: a retrospective observational study using population-based databases. Lancet Psychiatry 2018;5:824-35.

3. Cortese S, Adamo N, Del Giovane C, et al. Comparative efficacy and tolerability of medications for attention-deficit hyperactivity disorder in children, adolescents, and adults: a system- 
atic review and network meta-analysis. Lancet Psychiatry 2018; 5:727-38.

4. Moran LV, Ongur D, Hsu J, Castro VM, Perlis RH, Schneeweiss S. Psychosis with methylphenidate or amphetamine in patients with ADHD. N Engl J Med 2019;380:1128-38.

5. Gerstein HC, McMurray J, Holman RR. Real-world studies no substitute for RCTs in establishing efficacy. Lancet 2019; 393:210-1.

6. Man KK, Coghill D, Chan EW, et al. Methylphenidate and the risk of psychotic disorders and hallucinations in children and adolescents in a large health system. Transl Psychiatry 2016; 6(11):e956.

7. Bramness JG, Gundersen $\emptyset \mathrm{H}$, Guterstam J, et al. Amphet- amine-induced psychosis - a separate diagnostic entity or primary psychosis triggered in the vulnerable? BMC Psychiatry 2012;12:221.

8. Lichtenstein P, Halldner L, Zetterqvist J, et al. Medication for attention deficit-hyperactivity disorder and criminality. $\mathrm{N}$ Engl J Med 2012;367:2006-14.

9. Ross RG. Psychotic and manic-like symptoms during stimulant treatment of attention deficit hyperactivity disorder. Am J Psychiatry 2006;163:1149-52.

10. Cipriani A, Tomlinson A. Providing the most appropriate care to our individual patients. Evid Based Ment Health 2019;22:1-2. DOI: $10.1056 /$ NEJMe1900502

Copyright (C) 2019 Massachusetts Medical Society. 